\title{
Transmission Attributes of Periurban Malaria in Lusaka, Zambia, Precedent to the Integrated Vector Management Strategy: An Entomological Input
}

\author{
Emmanuel Chanda, ${ }^{1}$ Kumar S. Baboo, ${ }^{2}$ and Cecilia J. Shinondo ${ }^{3}$ \\ ${ }^{1}$ National Malaria Control Centre, Ministry of Health, Directorate of Public Health and Research, Chainama College Grounds, \\ P.O. Box 32509, 10101 Lusaka, Zambia \\ ${ }^{2}$ Department of Community Medicine, School of Medicine, University of Zambia, P.O. Box 50110, 10101 Lusaka, Zambia \\ ${ }^{3}$ Department of Biomedical Sciences, School of Medicine, University of Zambia, P.O. Box 50110, 10101 Lusaka, Zambia
}

Correspondence should be addressed to Emmanuel Chanda, emmanuel_chanda@yahoo.co.uk

Received 30 March 2012; Revised 31 May 2012; Accepted 13 August 2012

Academic Editor: Maria Aparecida Shikanai Yasuda

Copyright (C 2012 Emmanuel Chanda et al. This is an open access article distributed under the Creative Commons Attribution License, which permits unrestricted use, distribution, and reproduction in any medium, provided the original work is properly cited.

\begin{abstract}
Globalization and urbanization with their inherent developmental activities and ecological transformations impact on malaria epidemiology. Entomological factors involved in malaria transmission in periurban Lusaka were assessed prior to vector control reintroduction. Data was collected through standard entomological and epidemiological protocols and a pretested structured questionnaire. Larval habitats were characterized as transient (43\%), semipermanent (36\%), and permanent (21\%). Anopheles arabiensis and An. gambiae ss. were the only vectors identified. A shift in vector population was noted, with the later outnumbering the former. Plasmodium falciparum monoinfection rates were $25.6 \%$ (95\% CI: $20.9-30.7)(n=297)$. Parasitaemia was $31.8 \%$ (95\% CI: 23.2-42.2), 25.7\% (95\% CI: 13.5-41.3), and 23.3\% (95\% CI: 17.4-29.6) in under 5, 5 to 14, and above 15 age groups, respectively. Low knowledge levels on vector control tools with an average of 7 residents per household were also observed. This study confirmed a local malaria transmission paradigm. The epidemiology necessitated deployment of an integrated vector management strategy with intensified information education and communication.
\end{abstract}

\section{Introduction}

Malaria remains a serious global health problem, killing more than one million people per year. The global community has recently had many successes in malaria control. The number of malaria cases has fallen by more than $50 \%$ in 43 countries over the past decade [1]. A modeling analysis of malaria prevention activities in 34 African countries suggested that about 730,000 lives were saved between 2000 and 2010, with nearly three quarters of those since 2006 [2]. Funding commitments for malaria have increased nearly 15fold, from approximately US\$ 100 million in 2003 to nearly US\$ 1.6 billion in 2010; interest and commitment at global and country levels are very high [3]. However, the problem of malaria parasite transmission remains enormously grave in sub-Saharan Africa where at least 85 to $90 \%$ of deaths are attributable to the disease [4-7].
Malaria transmission is driven by a complex interaction of the vector, parasite, human host, and the environment, and is governed by different ecological and social determinants $[8,9]$. Globalization and urbanization with their inherent developmental activities and associated ecological transformations have a significant impact on malaria epidemiology $[10,11]$ and have invariably exacerbated the situation. Malaria transmission depends markedly on local environmental conditions and other compounding factors, that is, presence of drug-resistant parasites and insecticide resistant vectors [12, 13], environmental changes [14], economically driven human population increase and migration [15], poverty levels, climatic changes, natural disasters and political upheavals [16], adaptability of malaria vectors to changing environments $[17,18]$ and limited investment in research, drug discovery, and optimisation of malaria vector control programmes. 


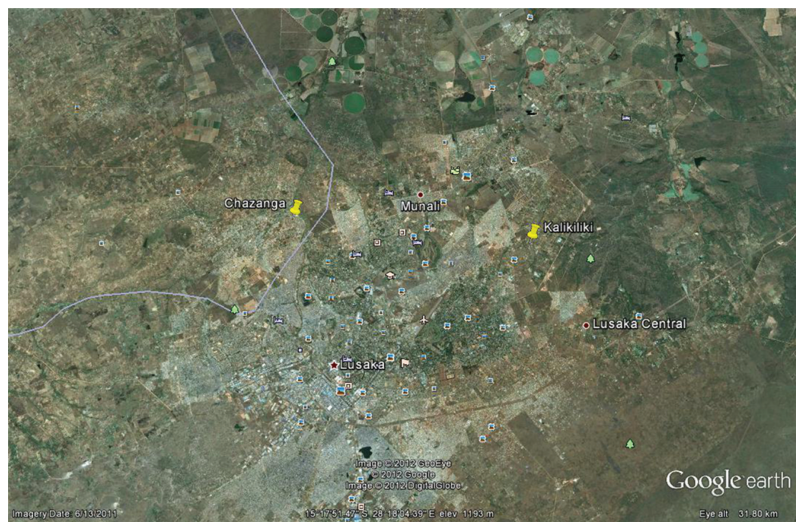

FIGURE 1: Map of greater Lusaka showing the periurban study site locations.

Transmission patterns and severity of malaria are influenced by the geographic attributes and the socioeconomic environment that vary significantly by city, season, and age group [19]. Accordingly, entomological profiles and clinical patterns are known to vary between urban, periurban, and rural environments [20]. Well-developed urban areas are mostly fringed by underdeveloped and inadequately serviced periurban areas experiencing the highest population growth rates [21] and often lacking infrastructure.

Malaria transmission in peri-urban areas is mostly ascribed to increased vector breeding created by the agricultural and construction activities, lack of drainage of surface water $[18,22,23]$, human vector contact due to poor housing and overcrowding [11], and low immunity in children under five and pregnant women, thus increasing the risk of severe disease [19]. In Zambia, between 1950 and early 1980s, vector control reduced malaria cases to a notifiable disease in most urban areas [24]. Ngandu and coworkers reported the resurgence of malaria cases in urban and peri-urban Lusaka [25]. In vivo sensitivity tests were also conducted with Plasmodium falciparum patients in Lusaka [26], but whether these infections were acquired in urban Lusaka itself or in rural areas was not clear.

Owing to malaria cases resurgence and paucity of entomological data, specific local investigations to appraise and confirm malaria transmission in peri-urban Lusaka were required before approaches to malaria vector control could be considered. We report here on malaria vectors, parasite prevalence rates in febrile patients and knowledge and attitudes of the community pertaining to malaria, precedent to the implementation of the integrated vector management (IVM) strategy.

\section{Materials and Methods}

2.1. Study Site. Zambia is a landlocked country in southern Africa with an estimated population of 13 million people, $45 \%$ are children below 15 years of age [27]. Malaria is endemic across the entire country with transmission peaks coinciding with the rainy season from November to April.
This study was conducted in two spatially segregated and randomly selected peri-urban locations of Lusaka district; Chazanga and Kalikiliki (Figure 1) during the cold-dry season from May to July 2003. The two sites have similar ecological characteristics and stretch out in an epidemiological zone characterized by low malaria transmission.

2.2. Mosquito Collections and Laboratory Processing. Mosquito larvae were collected from breeding sites using WHOstandard $250 \mathrm{~mL}$ dippers [28], transported to the insectary at the National Malaria Control Centre in Lusaka, and reared to adults while being fed on 1 part yeast and 2 parts dog biscuit. Adults were maintained on $10 \%$ sugar solution at $25 \pm 2^{\circ}$ centigrade temperature and $70-80 \%$ relative humidity.

Mosquito breeding sites were characterized into three different categories: transient, semipermanent, and permanent. Transient breeding site refers to temporal water collections, semi-permanent ones are those that would persist for a considerable period of time. Permanent breeding site refers to water bodies available throughout the year.

Adult mosquitoes were collected by the pyrethrum spray catch (PSC) between 06:00 hrs and 08:00 hrs in randomly selected households [28]. Anopheles mosquitoes were identified morphologically using standard keys for anophelines of southern Africa $[29,30]$ and to species by the polymerase chain reaction (PCR) molecular method of Scott et al. [31].

2.3. Parasitemia in Febrile Patients. Plasmodium falciparum infection was determined among febrile patients at health facilities in the study sites. Blood from randomly selected subjects who presented to the health center with febrile symptoms and consenting to participate was screened for parasite species and gametocytes by microscopy using 4\% Giemsa thick and thin blood smears for 30 minutes [32]. The age range of subjects was stratified into three age categories: 6 months $<5,5$ to $<15$, and 15 years and over. Participants with positive slide tests were offered free treatment with artemisinin-based combination therapy (ACT) according to Zambia national malaria control programme treatment policy guidelines [33]. 
TABLE 1: Entomological survey data.

\begin{tabular}{|c|c|c|c|c|}
\hline \multicolumn{5}{|c|}{ Breeding sites, larval densities and ratios } \\
\hline Habitat type & Transient & Semipermanent & Permanent & Total \\
\hline Abundance & $6(43 \%)$ & $5(36 \%)$ & $3(21 \%)$ & 14 \\
\hline Larvae collected & $1213(66 \%)$ & $508(28 \%)$ & $119(6 \%)$ & 1840 \\
\hline Anophelenes & $212(17.5 \%)$ & $161(31.7 \%)$ & $30(25 \%)$ & 403 \\
\hline Culicines & $1001(82.5 \%)$ & $347(68.3 \%)$ & $89(75 \%)$ & 1437 \\
\hline An-Cul ratio & 0.21 & 0.46 & 0.34 & \\
\hline Larvae $/ 250 \mathrm{~mL}$ & 70 & 45 & 20 & \\
\hline \multicolumn{5}{|c|}{ Vector molecular identification } \\
\hline & \multicolumn{2}{|c|}{ Kalikiliki } & \multicolumn{2}{|c|}{ Chazanga } \\
\hline An. gambiae ss. & \multicolumn{2}{|c|}{$11(58 \%)$} & \multicolumn{2}{|c|}{$7(37 \%)$} \\
\hline An. arabiensis & \multicolumn{2}{|c|}{0} & \multicolumn{2}{|c|}{$1(5 \%)$} \\
\hline
\end{tabular}

2.4. Knowledge and Attitudes. A pretested structured questionnaire was administered to 150 randomly selected respondents, tested for malaria, to determine community knowledge and attitudes as regards malaria, family demographic data, and possibility of malaria importation from rural areas.

2.5. Data Management and Statistical Analysis. Randomization was calculated for both study sites. Data was collected and entered in Excel spread sheets (Microsoft Corporation) and statistically analyzed by employing Epi Info version 3.2.2. The Chi-square $\left(\chi^{2}\right)$ test was used to determine the differences in parasite prevalence between age categories.

2.6. Ethical Consideration. Ethical approval for the research was granted by the University of Zambia Research Ethics Committee (Assurance number. FWA00000338 IRB00001131 of IOR G0000774). A freely administered informed consent was given to respondents and householders for participation in the study.

\section{Results}

3.1. Mosquito Collections. Of 1840 larvae collected, $66 \%$ (95\% CI: 65.7-68.1) were from transient (gardens and abandoned building foundations), 28\% (95\% CI: 25.6-29.6) semipermanent (abandoned shallow wells and ditches that followed in the wake excavations for building sand or quarrying) and 6\% (95\% CI: 5.4-7.7) permanent water bodies (perennial streams and dams) (Figure 2). Anophelines accounted for only $21.9 \%$ (95\% CI: 20.1-23.9). The density of Anopheles larvae was comparatively higher in semipermanent $(31.7 \%)$ followed by the permanent (25\%) and transient habitats (17.5\%) (Figure 2 and Table 1).

Anophelines constituted 12.83\% (95\% CI: 8.7-17.9) of the 203 adult mosquitoes collected (Table 1). The mosquito male-to-female ratios and densities per room was 0.59 to 0.26 and 1.7 to 15 for Anopheles and Culex, respectively. A total of 30 An. gambiae ss. were subjected to molecular assays including those reared from larvae. 11 could not amplify a PCR product. All specimens from Kalikiliki $(n=11)$ and
Chazanga $(n=7)$, amplified for An. gambiae ss. and only 1 from Chazanga amplified for An. arabiensis (Figures 3 and 4).

3.2. Parasitemia in Febrile Patients. A total of 297 randomly selected febrile patients were recruited into the study (Table 2). The age of the subjects ranged from 6 months to $60+$ years. Seventy-six (25.6\%) were positive for malaria parasites with $100 \%$ Plasmodium falciparum parasite monoinfection. Among the positive slides, 75 (98.7\%) exhibited ring form trophozoites and only 1 (1.3\%) showed gametocytaemia. The parasitemia in febrile patients per age group was $31.8 \%$ (95\% CI: $23.2-42.2$ ) for the $0-4$ years group, $25.7 \%$ (95\% CI: $13.5-41.3$ ) for 5 to 15 years, and $23.3 \%$ (95\% CI: 17.4-29.6) for the 15 years and over $(P>0.05)$.

3.3. Knowledge and Attitudes. Of the 150 respondents $18 \%$ (95\% CI: $12.4-24.6)$ were male and 82\% (95\% CI: 75.4-87.3) were female. The mean age was 29.9 with a range of 18 to 53 years. Forty-eight percent exhibited good knowledge of malaria as a disease. Sixty-three percent were knowledgeable about malaria transmission. Seventy-nine per cent were conversant with causes, signs and symptoms. Sixty-two per cent showed awareness of what to do when they suspected malaria and only forty-six per cent were knowledgeable about vector control interventions. Family demographic data showed an average of seven residents with at least one child under five years per household. Eighty-one per cent of respondents had no history of travel outside Lusaka. There was positive association between knowledge and malaria prevalence in periurban Lusaka $(P<0.05)$.

\section{Discussion}

The malaria vectorial system in Zambia comprises of $A n$. gambiae ss., An. arabiensis, and An. funestus [34, 35], with great divergence in their malaria transmission potential, spatial segregation, and temporal heterogeneity [36, 37]. The pioneering malaria control efforts in the country $[38,39]$ stimulated unprecedented enthuse in entomological studies [36, 40-44]. Recent studies have demonstrated the presence of An. nili, An. funestus-like, and An. rivulorum although 
Abundance of breeding sites, collected larvae, and

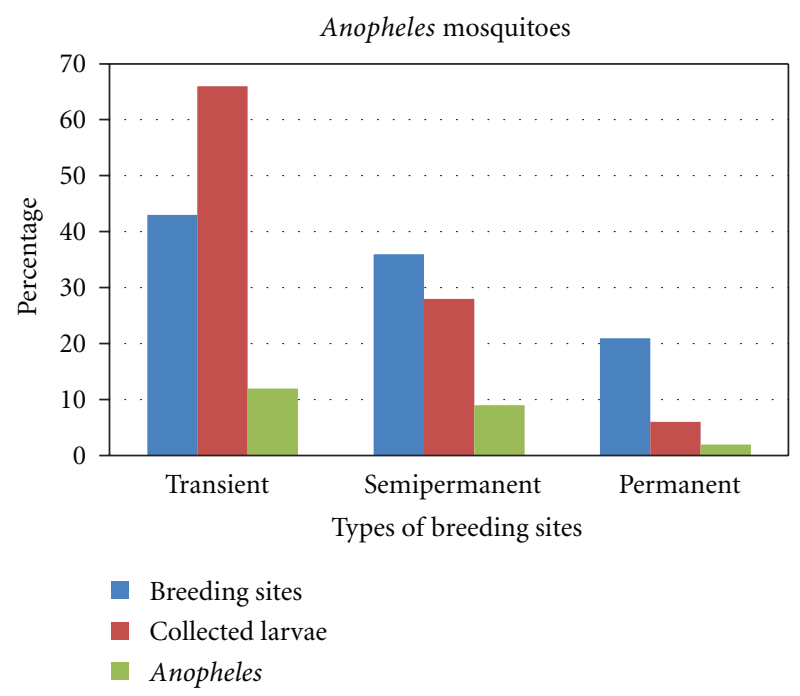

FIGURE 2: Abundance of breeding sites, collected larvae, and Anopheles mosquitoes.

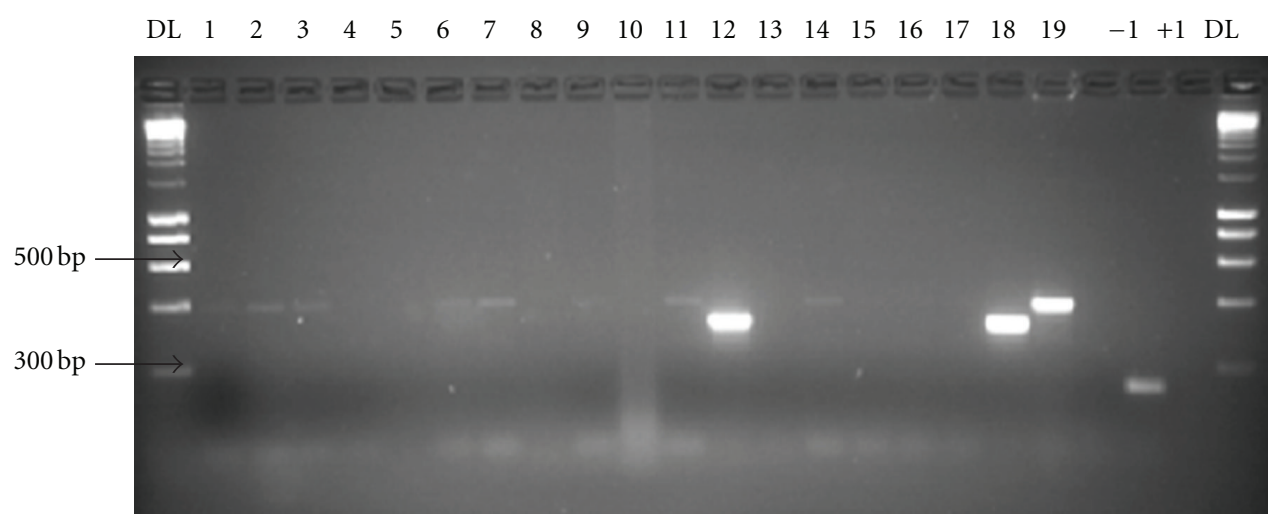

Figure 3: DNA bands produced by ribosomal DNA-polymerase chain reaction (PCR) amplification from the different species in the Anopheles gambiae complex from Chazanga. DL: 1-kp DNA ladder size standards, +1: positive control (A. arabiensis), -1: negative control. The sample DNA in each of the lanes was as follows: 1, 2, 3, 6, 7, 11, and 14 were amplified for A. gambiae ss. (390 bp). 12 was amplified for A. arabiensis (315 bp).

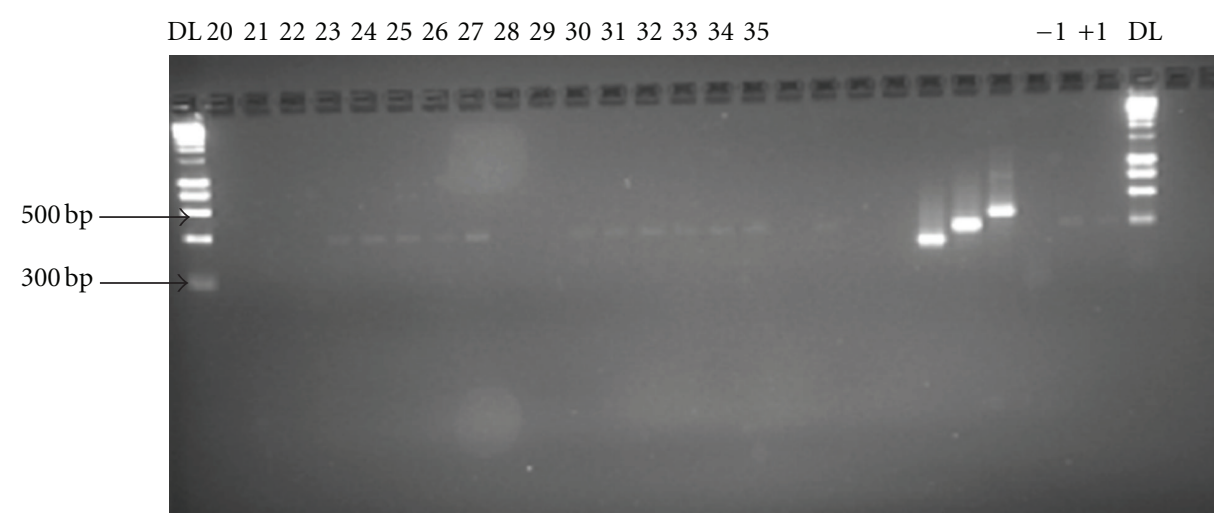

FIGURE 4: DNA bands produced by ribosomal DNA-polymerase chain reaction (PCR) amplification from the different species in the Anopheles gambiae complex from Kalikiliki. DL: 1-kbp DNA ladder size standards, +1: positive control (A. arabiensis), and -1: negative control. The sample DNA in each of the lanes was as follows: 23, 24, 25, 26, 27, 30, 31, 32, 33, 34, and 35 were amplified for A. gambiae ss. (390 bp). 
TABle 2: Parasitological survey data.

\begin{tabular}{|c|c|c|c|c|}
\hline \multicolumn{5}{|c|}{ Parasitemia in febrile patients by age and sex } \\
\hline Age group & $0-4$ yrs & $5-15$ yrs & $>15$ yrs & Total \\
\hline \multirow{2}{*}{ Number surveyed } & $52^{*}$ & $14^{*}$ & $63^{*}$ & 129 \\
\hline & $38^{\dagger}$ & $21^{\dagger}$ & $109^{\dagger}$ & 168 \\
\hline Frequency (age) & $27(30 \%)$ & $9(25.7 \%)$ & $40(23.3 \%)$ & 76 \\
\hline \multirow{2}{*}{ Frequency (sex) } & $15^{*}$ & $4^{*}$ & $26^{*}$ & 45 \\
\hline & $12^{\dagger}$ & $5^{\dagger}$ & $14^{\dagger}$ & 31 \\
\hline \multicolumn{5}{|c|}{ Parasite densities by age } \\
\hline $1-10 / 100$ O.I & $25(62.5 \%)$ & $3(33.3 \%)$ & $15(55.6 \%)$ & 43 \\
\hline $11-100 / 100$ O.I & $9(19.0 \%)$ & $2(22.2 \%)$ & $4(17.4 \%)$ & 15 \\
\hline 10/O.I & $3(7.5 \%)$ & $1(11.1 \%)$ & $3(7.5 \%)$ & 7 \\
\hline$>10 /$ O.I & $3(7.5 \%)$ & $1(11.1 \%)$ & $5(18.5 \%)$ & 11 \\
\hline
\end{tabular}

O.I: oil immersion field, ${ }^{\dagger}$ female, and $*$ male.

their role in malaria transmission in Zambia is yet to be established [45].

Urban areas are perceived not to support significant levels of malaria transmission [18]. In this study, three kinds of mosquito breeding habitats: transient, semipermanent, and permanent were characterized with appreciable spatial heterogeneity (Figure 2). An. gambiae is known to exploit small open temporal habitats with less predation, increased warmth, and more algae [46]. However, more Anopheles larvae were collected from semipermanent habitats than from permanent and transient habitats (Figure 2 and Table 1). This could explain the role of urban development related activities in supporting high malaria transmission levels as observed in peri-urban Lusaka.

While formal urban development typically reduces mosquito densities, informal urbanization has been shown to alter the vector species composition within the An. gambiae complex in sub-Saharan Africa, [47]. To illustrate, earlier studies conducted in Zambia indicated $100 \%$ An. arabiensis $[35,36]$. Nevertheless, the profound demographic and extensive environmental changes that have followed in the wake of urbanization have changed the stratification of the vectors. This study demonstrates coexistence of An. gambiae ss. and An. arabiensis with the former greatly outnumbering the later in complete absence of An. funestus. Notably, the predominance of An. gambiae ss. validates the premise that informal urban development does transform vector species composition.

The presence of An. arabiensis, a species that is typically difficulty to control by IRS and ITNs, and the predominance of the An. gambiae ss. which is characteristically amenable to control by IRS and ITNs [48] could have implications for the malaria control programme. The sympatric-existence of these vectors demonstrates the need for an integrated approach for malaria vector control. This study was characterized by low number of mosquito collections due to the unfavorable prevailing environmental conditions during the cold season, lack of data on chromosomal forms of $A n$. gambiae ss. and transmission determining parameters, that is, vector infectivity. However, early entomological work in Zambia reported a sporozoite rate of $1.4 \%$ in An. arabiensis in Lusaka [44]. Notably, there is still a clear paucity of data on malaria vector bionomics in the country.

Malaria had been known to be hyperendemic in hot riverine valleys with perennial transmission, meso-to hypoendemic on plateaus, and hypo-endemic in urban areas of Zambia [49]. Between 1969 and 2000, parasite rates ranged from 2.0 to $26.4 \%$ across the country [39], with parasite species of $86.8 \%$ P. falciparum and $13.2 \%$ P. malariae in Ndola rural [50]. By 1999, parasite species was over $97 \%$ $P$. falciparum [49]. These findings are corroborated in this study with $25.3 \%$ parasitaemia among febrile patients with $100 \%$ P. falciparum monoinfections. This upsurge of frequency of febrile malaria was further aggravated by the development of chloroquine resistance [51]. Deployment of effective control tools has transformed the epidemiological profile from countrywide high endemicity to three distinct epidemiological strata: very low transmission and parasite prevalence of $<1 \%$, low transmission $(<10 \%)$, and persistent high transmission $(>20 \%)$.

The prevalence rate of malaria in children under five years is dependent on the intensity of transmission and declines with age as immunity develops and is thus a good indicator of a recent transmission of malaria [52]. The highest prevalence of malaria in Zambia occurs in this age group across the country [49]. In this study, frequency of febrile malaria was highest $(31.8 \%)$ in the $0-4$ years age group and lowest $(23.3 \%)$ in the 15 years and above group. There was no significant difference in parasitaemia in febrile patients of the three age categories $(P>0.05)$ suggesting a nonimmune population and an area of low transmission. The above $10 \%$ parasitaemia observed in children under 5 years of age confirmed that malaria had again become endemic in peri-urban Lusaka [18].

The knowledge and attitudes survey indicated the need for intensified information, education and communication (IEC) on malaria and its prevention. The $46 \%$ knowledge level on vector control interventions indicated a weakness in individual efforts to prevent the disease. Population expansion and its health impact has been epitomized by subSaharan Africa. In many malaria endemic countries, including Zambia, the population has doubled in the past two 
decades, thus greatly increasing the absolute numbers of those at risk [53]. Accordingly, the peri-urban settlements experience the highest population growth rates [11]. This was demonstrated in peri-urban Lusaka where family demographic data showed an average of seven residents with at least one child less than five years per household. Thus, suggesting that congestion in households was probably one of the factors contributing to the increased transmission of malaria in these settings.

It has equally been established that human migration contributes markedly to malaria transmission [54]. In areas of endemicity, encroaching transmission has been demonstrated in areas previously free of transmission and local transmission has been conclusively demonstrated in many African cities $[55,56]$. These findings are corroborated in this study which confirmed local transmission in Lusaka as $80 \%$ subjects with definitively diagnosed malaria had no history of travel. It was established that there is no significant contribution of migration towards malaria transmission in periurban Lusaka $(P>0.05)$. Local transmission of malaria was further strongly inferred by high parasitaemia in children under the age of five and the presence of gametocyte bearers and efficient vectors in the community that perpetuated the transmission cycle. Congestion in households together with the appreciably low levels of knowledge on control and prevention compounded the situation.

The pragmatic data reported on here was an essential prerequisite of evidence-based and effective vector control efforts. The high malaria infection rates in peri-urban Lusaka could be ascribed to the definitively demonstrated local transmission. This necessitated the institution of appropriate control strategies based on the prevailing transmission paradigm. The presence of An. gambiae complex species and characterization of their breeding attributes required an integrated vector management (IVM) approach to effectively control transmission. It is noteworthy, that this preintervention study had limitations as the surveys were conducted during the dry season which markedly influenced the malaria vector and parasite populations.

Clearly, the malaria epidemiology in peri-urban Lusaka required an integrated approach involving IRS and ITNs against the adults and larval source management (LSM) against the aquatic stages. Information education and communication (IEC) to increase awareness and knowledge about malaria vector control needed to be intensified. Following this study, IVM was introduced in Lusaka with IRS and ITNs as main thrust interventions and IEC has been strengthened [57]. This has reduced malaria parasite rates to appreciably minimal levels $(<1 \%)$ [58]. To clear the residual transmission, LSM is being implemented in Lusaka. While monitoring and evaluation of vector control interventions has been strengthened [45], it is imperative that a comprehensive entomological and epidemiological surveillance system is established to detect any increase in the malaria case load.

\section{Conflict of Interests}

The authors declare that they have no conflict interests.

\section{Authors' Contribution}

E. Chanda: codesigned the study, collected and analysed the data, and drafted the paper. K. S. Baboo: critically reviewed the manuscript. C. J. Shinondo codesigned the study, guided in data analysis and interpretation and contributed to the drafting of the paper and critically evaluated it. All authors read and approved the final paper.

\section{Acknowledgments}

The aurhors thank all Ministry of Health staff from the Health Centres and National Malaria Control Centre who participated in the study. They thank Dr. John Govere and the two anonymous reviewers for their useful comments on the paper. They also thank the Lusaka District Health Office for authority to work in Health facilities and the National Institute for Communicable Diseases, South Africa, for analyzing the malaria vector samples. The work was supported by the United States Agency for International Development (USAID).

\section{References}

[1] WHO, "World Malaria Report," Tech. Rep., World Health Organization, Geneva, Switzerland, 2012.

[2] T. P. Eisele, D. A. Larsen, N. Walker et al., "Estimates of child deaths prevented from malaria prevention scale-up in Africa 2001-2010," Malaria Journal, vol. 11, article 93, 2012.

[3] T. P. Eisele, D. Larsen, and R. W. Steketee, "Protective efficacy of interventions for preventing malaria mortality in children in Plasmodium falciparum endemic areas," International Journal of Epidemiology, vol. 39, pp. i88-101, 2010.

[4] J. G. Breman, A. Egan, and G. T. Keusch, "The intolerable burden of malaria: a new look at the numbers," American Journal of Tropical Medicine and Hygiene, vol. 64, no. 1-2, pp. 4-7, 2001.

[5] R. W. Snow, C. A. Guerra, A. M. Noor, H. Y. Myint, and S. I. Hay, "The global distribution of clinical episodes of Plasmodium falciparum malaria," Nature, vol. 434, no. 7030, pp. 214217, 2005.

[6] C. A. Guerra, R. W. Snow, and S. I. Hay, "Defining the global spatial limits of malaria transmission in 2005," Advances in Parasitology, vol. 62, pp. 157-179, 2006.

[7] J. G. Breman, M. S. Alilio, and A. Mills, "Conquering the intolerable burden of malaria: what's new, what's needed: a summary," American Journal of Tropical Medicine and Hygiene, vol. 71, no. 2, pp. 1-15, 2004.

[8] S. I. Hay, "Remotely sensed surrogates of meteorological data for the study of the distribution and abundance of arthropod vectors of disease," Annals of Tropical Medicine and Parasitology, vol. 90, no. 1, pp. 1-19, 1996.

[9] A. Daash, A. Srivastava, B. N. Nagpal, R. Saxena, and S. K. Gupta, "Geographical information system (GIS) in decision support to control malaria-a case study of Koraput district in Orissa, India," Journal of Vector Borne Diseases, vol. 46, no. 1, pp. 72-74, 2009.

[10] S. I. Hay, C. A. Guerra, A. J. Tatem, P. M. Atkinson, and R. W. Snow, "Urbanization, malaria transmission and disease burden in Africa," Nature Reviews Microbiology, vol. 3, no. 1, pp. 81-90, 2005. 
[11] J. Keiser, J. Utzinger, M. Caldas De Castro, T. A. Smith, M. Tanner, and B. H. Singer, "Urbanization in sub-Saharan Africa and implication for malaria control," American Journal of Tropical Medicine and Hygiene, vol. 71, no. 2, pp. 118-127, 2004.

[12] K. I. Barnes, F. Little, A. Mabuza et al., "Increased gametocytemia after treatment: an early parasitological indicator of emerging sulfadoxine-pyrimethamine resistance in falciparum malaria," Journal of Infectious Diseases, vol. 197, no. 11, pp. 1605-1613, 2008.

[13] J. Hemingway and H. Ranson, "Insecticide resistance in insect vectors of human disease," Annual Review of Entomology, vol. 45, pp. 371-391, 2000.

[14] J. Keiser, M. C. De Castro, M. F. Maltese et al., "Effect of irrigation and large dams on the burden of malaria on a global and regional scale," American Journal of Tropical Medicine and Hygiene, vol. 72, no. 4, pp. 392-406, 2005.

[15] P. Martens and L. Hall, "Malaria on the move: human population movement and malaria transmission," Emerging Infectious Diseases, vol. 6, no. 2, pp. 103-109, 2000.

[16] B. Greenwood and T. Mutabingwa, "Malaria in 2002," Nature, vol. 415, no. 6872, pp. 670-672, 2002.

[17] W. A. Chinery, "Effects of ecological changes on the malaria vectors Anopheles funestus and the Anopheles gambiae complex of mosquitoes in Accra, Ghana," Journal of Tropical Medicine and Hygiene, vol. 87, no. 2, pp. 75-81, 1984.

[18] W. Mc Wilsons, P. Billing, D. Bendahmane, and P. Wijeyanratne, "Malaria in rural and peri-urban areas in Sub-Saharan Africa," Environmental Health Project Activity Report 71, 1999.

[19] S. J. Wang, C. Lengeler, T. A. Smith et al., "Rapid urban malaria appraisal (RUMA) in sub-Saharan Africa," Malaria Journal, vol. 4, article 40, 2005.

[20] D. Modiano, B. S. Sirima, A. Sawadogo et al., "Severe malaria in Burkina Faso: urban and rural environment," Parassitologia, vol. 41, no. 1-3, pp. 251-254, 1999.

[21] A. B. Knudsen and R. Slooff, "Vector-borne disease problems in rapid urbanization: new approaches to vector control," Bulletin of the World Health Organization, vol. 70, no. 1, pp. 1-6, 1992.

[22] Y. A. Afrane, E. Klinkenberg, P. Drechsel, K. Owusu-Daaku, R. Garms, and T. Kruppa, "Does irrigated urban agriculture influence the transmission of malaria in the city of Kumasi, Ghana?" Acta Tropica, vol. 89, no. 2, pp. 125-134, 2004.

[23] V. Robert, H. P. Awono-Ambene, and J. Thioulouse, "Ecology of Larval mosquitoes, with special reference to Anopheles arabiensis (Diptera: Culcidae) in Market-Garden Wells in Urban Dakar, Senegal," Journal of Medical Entomology, vol. 35, no. 6, pp. 948-955, 1998.

[24] L. M. Barat, B. Himonga, S. Nkunika et al., "A systematic approach to the development of a rational malaria treatment policy in Zambia," Tropical Medicine and International Health, vol. 3, no. 7, pp. 535-542, 1998.

[25] N. Ng'andu, T. E. Watts, J. R. Wray, C. Chela, and B. Zulu, "Some risk factors for transmission of malaria in a population where control measures were applied in Zambia," East African Medical Journal, vol. 66, no. 11, pp. 728-737, 1989.

[26] G. J. Blom, K. S. Baboo, U. H. Athale, and T. S. Van Der Werf, "Plasmodium falciparum malaria in vivo drug sensitivity in Lusaka, Zambia," Central African Journal of Medicine, vol. 41, no. 1, pp. 6-10, 1995.

[27] Central Statistical Office, "Zambia National Census Report," Tech. Rep., 2000.
[28] WHO, Manual on Practical Entomology in Malaria. PartII: Methods and Techniques, 1975.

[29] M. T. Gillies and M. Coetzee, A Supplement to the Anophelinae of Africa South of the Sahara (Afro-Tropical Region), vol. 55, South African Institute for Medical Research, Johannesburg, South Africa, 1987.

[30] M. T. Gillies and B. A. De Meillon, The Anophelinae of Africa South of the Sahara (Ethiopian Zoogeographical Region), vol. 54, South African Institute for Medical Research, Johannesburg, 2nd edition, 1968.

[31] J. A. Scott, W. G. Brogdon, and F. H. Collins, "Identification of single specimens of the Anopheles gambiae complex by the polymerase chain reaction," American Journal of Tropical Medicine and Hygiene, vol. 49, no. 4, pp. 520-529, 1993.

[32] World Heath Organization, "Basic laboratory methods in medical parasitology," in Basic Malaria Microscopy, Part I, WHO, Geneva, Switzerland, 1991.

[33] MoH, National Malaria Strategic Plan 2006-2011: A Road Map for RBM Impact in Zambia, Ministry of Health, Lusaka, Zambia, 2006.

[34] G. Macdonald, The Epidemiology and Control of Malaria, Oxford University Press, London, UK, 1957.

[35] W. R. Bransby-Williams, "House catches of adult Anopheles gambiae species B in two areas of Zambia," East African Medical Journal, vol. 56, no. 11, pp. 557-561, 1979.

[36] A. J. Shelley, "Observations on the behaviour of Anopheles gambiae sp. B in Kambole village in the Zambesi valley, Zambia," Annals of Tropical Medicine and Parasitology, vol. 67, no. 2, pp. 237-248, 1973.

[37] D. J. Rogers, S. E. Randolph, R. W. Snow, and S. I. Hay, "Satellite imagery in the study and forecast of malaria," Nature, vol. 415, no. 6872, pp. 710-715, 2002.

[38] M. Watson, African Highway: the Battle for Health in Central Africa, John Murray, London, UK, 1953.

[39] J. Utzinger, Y. Tozan, and B. H. Singer, "Efficacy and costeffectiveness of environmental management for malaria control," Tropical Medicine and International Health, vol. 6, no. 9, pp. 677-687, 2001.

[40] B. De Meillon, "A note on An. gambiae and An. funestus in Northern Rhodesia," Entomolgoical Studies, article 7, p. 306, 1937.

[41] P. C. G. Adams, "Some observations on the flight of stained anophelines at Nkana, Northern Rhodesia," Annals of Tropical Medicine and Parasitology, pp. 34-35, 1940.

[42] D. Pielou, "Anopheline mosquitoes breeding in fish dams, pools, and streams in Northern Rhodesia," Proceedings of the Royal Entomological Society of London, vol. 22, pp. 18-23, 1947.

[43] H. E. Paterson, "The species, species control and antimalarial spraying campaigns, implications of recent work on the An. gambiae complex," South African Journal of Medical Science, vol. 28, pp. 33-44, 1963.

[44] A. Zahar, "Vector bionomics in the epidemiology and control of malaria," Part I, the WHO African Region \& the Southern WHO Eastern Mediterranean Region. Section III., (Equatorial Africa) (Southern Africa) WHO/VBC/85.2, World Health Organization, Geneva, Switzerlands.

[45] E. Chanda, J. Hemingway, I. Kleinschmidt et al., "Insecticide resistance and the future of malaria control in Zambia," PLoS One, vol. 6, no. 9, Article ID e24336, 2011.

[46] J. E. Gimmg, M. Ombok, L. Kamau, and W. A. Hawley, "Characteristics of larval anopheline (Diptera: Culicidae) habitats in western Kenya," Journal of Medical Entomology, vol. 38, no. 2, pp. 282-288, 2001. 
[47] V. A. Wagbatsoma and O. Ogbeide, "Towards malaria control in Nigeria: a qualitative study on the population of mosquitoes," Journal of the Royal Society of Health, vol. 115, no. 6, pp. 363-365, 1995.

[48] S. W. Lindsay, L. Parson, and C. J. Thomas, "Mapping the ranges and relative abundance of the two principal African malaria vectors, Anopheles gambiae sensu stricto and An. arabiensis, using climate data," Proceedings of the Royal Society B, vol. 265, no. 1399, pp. 847-854, 1998.

[49] MoH, National Malaria Situation Analysis, Ministry of Health, Lusaka, Zambia, 2000.

[50] R. W. Wenlock, "The incidence of Plasmodium parasites in rural Zambia," East African Medical Journal, vol. 55, no. 6, pp. 268-276, 1978.

[51] N. Sipilanyambe, J. L. Simon, P. Chanda, P. Olumese, R. W. Snow, and D. H. Hamer, "From chloroquine to artemetherlumefantrine: the process of drug policy change in Zambia," Malaria Journal, vol. 7, article 25, 2008.

[52] L. J. Bruce-Chwatt, Essential Malariology, John Willey \& Sons, New York, NY, USA, 2nd edition, 1985.

[53] United Nations Population Fund, State of the World Population: Sub-Saharan Africa, UNPF, Geneva, Switzerland, 1996.

[54] P. Muentener, P. Schlagenhauf, and R. Steffen, "Imported malaria (1985-95): trends and perspectives," Bulletin of the World Health Organization, vol. 77, no. 7, pp. 560-566, 1999.

[55] K. A. Lindblade, E. D. Walker, A. W. Onapa, J. Katungu, and M. L. Wilson, "Highland malaria in Uganda: prospective analysis of an epidemic associated with El Nino," Transactions of the Royal Society of Tropical Medicine and Hygiene, vol. 93, no. 5, pp. 480-487, 1999.

[56] M. Albonico, F. De Giorgi, J. Razanakolona et al., "Control of epidemic malaria on the Highlands of Madagascar," Parassitologia, vol. 41, no. 1-3, pp. 373-376, 1999.

[57] E. Chanda, F. Masaninga, M. Coleman et al., "Integrated vector management: the Zambian experience," Malaria Journal, vol. 7, article no. 164, 2008.

[58] MoH, "Zambia National Malaria Indicator," Survey Report 2008, Ministry of Health, Lusaka, Zambia, 2008. 


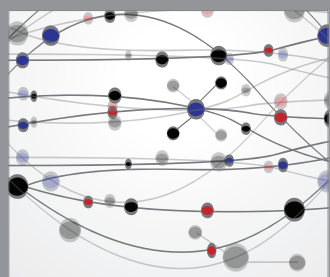

The Scientific World Journal
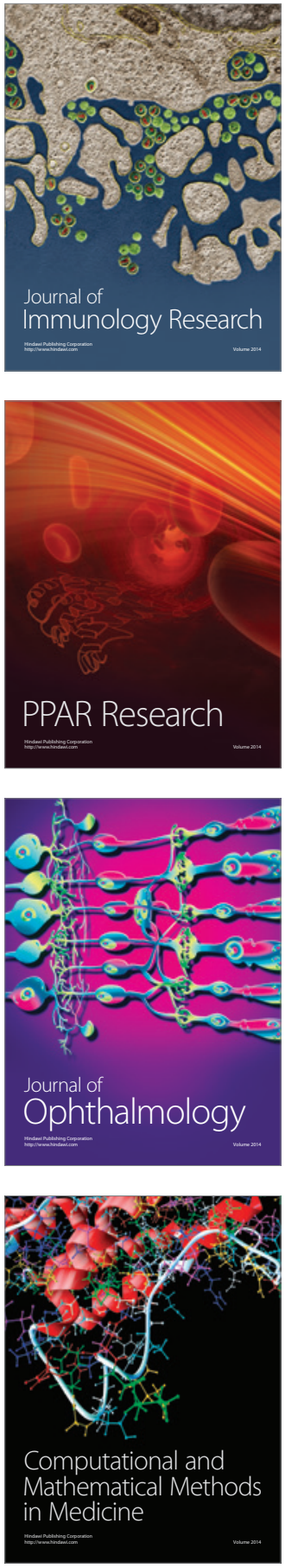

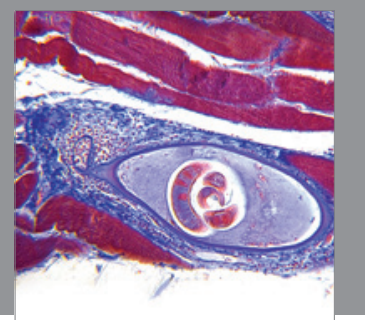

Gastroenterology

Research and Practice
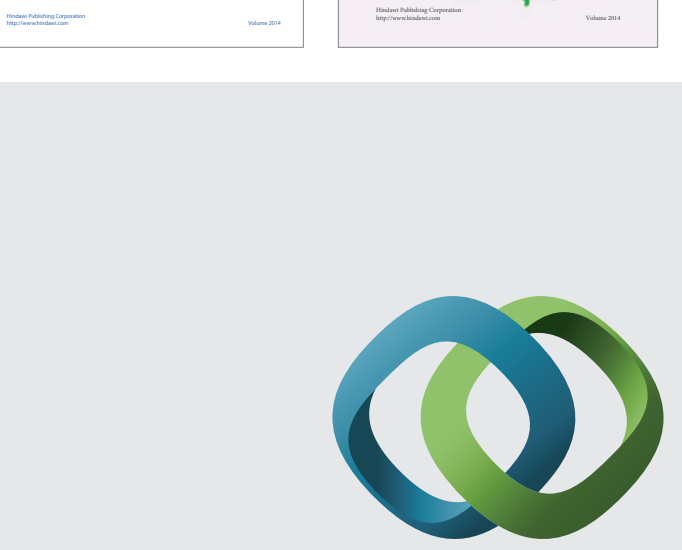

\section{Hindawi}

Submit your manuscripts at

http://www.hindawi.com
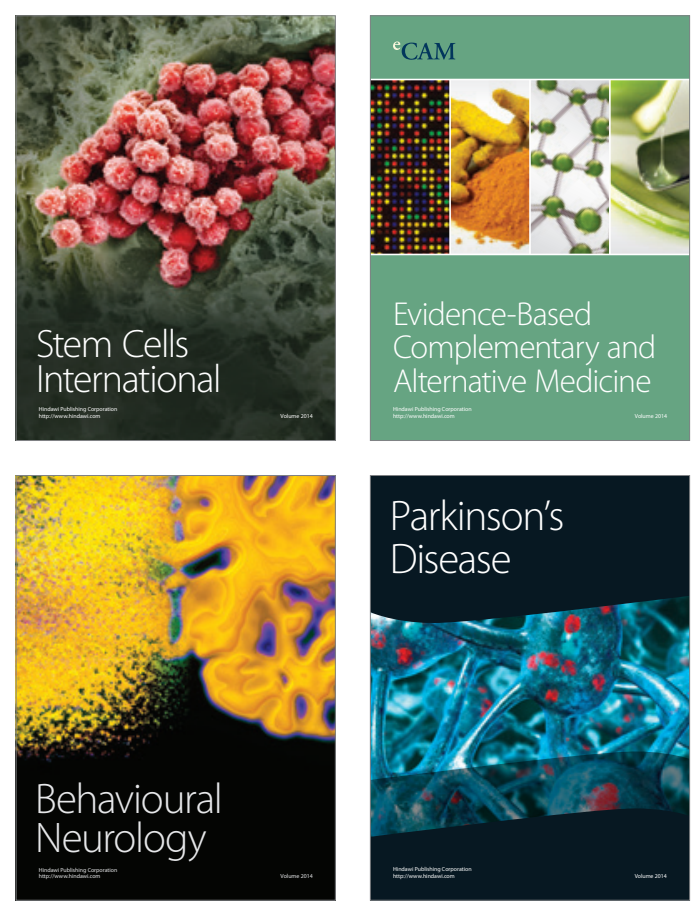

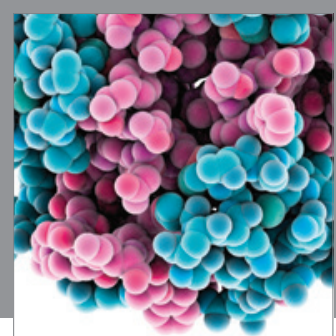

Journal of
Diabetes Research

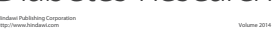

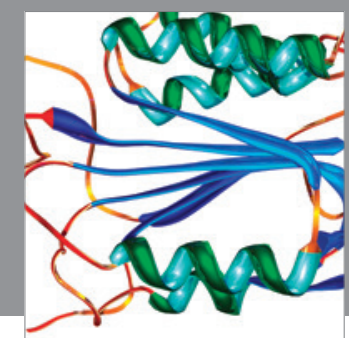

Disease Markers
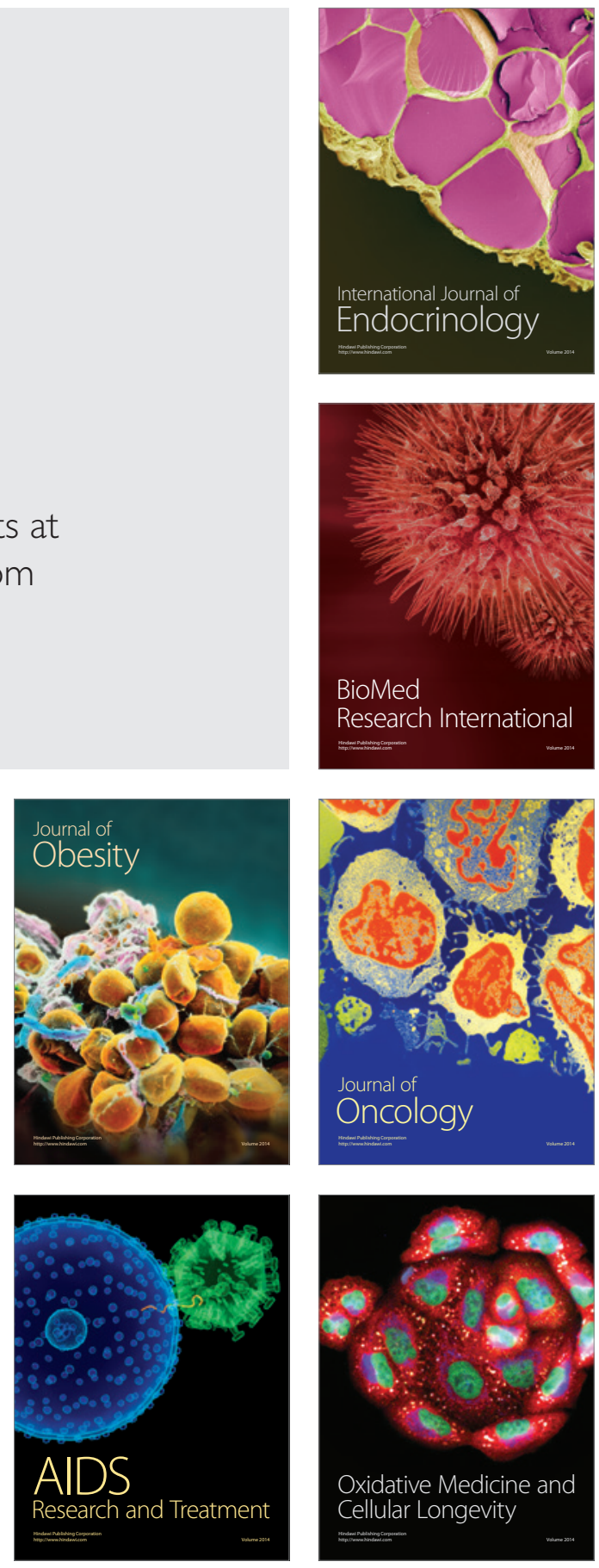